\title{
The limit of spin lifetime in solid-state electronic spins
}

\author{
${ }^{1}$ Alessandro Lungh: ${ }^{*}$ and ${ }^{1}$ Stefano Sanvito \\ ${ }^{1}$ School of Physics, AMBER and CRANN, Trinity College, Dublin 2, Ireland
}

\begin{abstract}
The development of spin qubits for quantum technologies requires their protection from the main source of finite-temperature decoherence: atomic vibrations. Here we eliminate one of the main barriers to the progress in this field by providing a complete first-principles picture of spin relaxation that includes up to two-phonon processes. Our method is based on machine learning and electronic structure theory and makes the prediction of spin lifetime in realistic systems feasible. We study a prototypical vanadium-based molecular qubit and reveal that the spin lifetime at high temperature is limited by Raman processes due to a small number of THz intra-molecular vibrations. These findings effectively change the conventional understanding of spin relaxation in this class of materials and open new avenues for the rational design of long-living spin systems.
\end{abstract}

The observation of matter in a coherent superposition of quantum states is a core fingerprint of quantum mechanics. While the laws of Physics allow any object, regardless of its size, to be prepared in such state, the unwanted interaction of a quantum system with a large number of other degrees of freedom causes the destruction of coherence and restore the classical picture of the world [1]. Spins, either nuclear or electronic, are naturally loosely coupled to other degrees of freedom. The coherent control of their quantum states has been achieved in several physical systems, including both solid-state semiconductors [2] and molecules [3]. Several strategies designed to preserve the coherence time, $T_{2}$, from the effect of spurious magnetic interactions have been successful implemented [4, 5]. However, spins also inevitably interact with the atomic motion and relax to a non-coherent thermal equilibrium state on a time scale, $\tau$, also called $T_{1}$ or spin-lattice relaxation time. This is the ultimate limit for the coherence time.

Here we provide a first-principles description of these relaxation processes and show the limits that solid-state vibrations pose to the spin lifetime. Despite the central importance of spin-lattice relaxation for a broad range of disciplines, such as magnetism [6, quantum computation [7] and magnetic resonance [8, its parameterfree first-principles theoretical description is almost unexplored due to the associated daunting computational requirements 7, 9 11. In this work we solve the challenge by designing a machine-learning-accelerated 12, 13 firstprinciples strategy able to account for both one- and twophonon spin relaxation processes. Multi-phonon contributions have so far been beyond the reach of computational methods and their inclusion provides a complete picture of spin relaxation [11. We anticipate that these results will enable new routes for the design of long-living spin systems.

We will use as a test case the study of the $\mathrm{VO}(\text { acac })_{2}$ molecular qubit embedded in a solid-state crystal [14].

\footnotetext{
* lunghia@tcd.ie
}

This $\mathrm{V}^{4+}$-based molecule bears both an electronic, $\overrightarrow{\mathbf{S}}$, and a nuclear spin, $\overrightarrow{\mathbf{I}}$, and represents a typical building block for quantum computing platforms [15-17].

In the absence of others spins from the ones explicitly considered, a situation also known as magnetic diluted condition, the spin dynamics of a $\mathrm{VO}(\mathrm{acac})_{2}$ unit is driven by the spin Hamiltonian,

$$
\hat{H}_{\mathbf{s}}=\beta_{e} \overrightarrow{\mathbf{B}} \cdot \mathbf{g}_{\mathbf{e}} \cdot \overrightarrow{\mathbf{S}}+\beta_{n} \overrightarrow{\mathbf{B}} \cdot \mathbf{g}_{\mathbf{n}} \cdot \overrightarrow{\mathbf{I}}+\overrightarrow{\mathbf{S}} \cdot \mathbf{A} \cdot \overrightarrow{\mathbf{I}}
$$

where the electronic $(S=1 / 2)$ and nuclear $(I=7 / 2)$ spins of ${ }^{51} \mathrm{~V}$ interact with each other through the hyperfine tensor, $\mathbf{A}$, and with the external magnetic field, $\overrightarrow{\mathbf{B}}$, through the electronic (nuclear) gyromagnetic tensor, $\beta_{e} \mathbf{g}_{\mathbf{e}}\left(\beta_{n} \mathbf{g}_{\mathbf{n}}\right)$. As schematically shown in Fig. 11A, the Zeeman and hyperfine interactions split the spin spectrum, $\omega$, in the $10^{-2}-10 \mathrm{~cm}^{-1}$ range, depending on the size of the external field. For energies comparable to $k_{\mathrm{B}} T$, where $k_{\mathrm{B}}$ is the Boltzman constant, only these states will be populated, as the first excited electronic state is more than $10,000 \mathrm{~cm}^{-1}$ higher in energy.

In the absence of external perturbations, the state of $\overrightarrow{\mathbf{S}}$ and $\overrightarrow{\mathbf{I}}$, described by the spin-density matrix, $\hat{\rho}$, would evolve coherently in time according to the Liouville equation, $i \hbar(d \hat{\rho} / d t)=\left[\hat{H}_{\mathrm{s}}, \hat{\rho}\right]$. However, in a typical solidstate environment, such as the $\mathrm{VO}(\mathrm{acac})_{2}$ molecular crystal, the spin lifetime has a finite value due to the coupling of spins with phonons $\hat{Q}_{\alpha \mathbf{q}}$, namely with the oscillations of the atoms' positions with frequency, $\omega_{\alpha \mathbf{q}}$, and reciprocal lattice vector q. According to Redfield theory [18, the spin-relaxation rate, $\tau^{-1}$, due to the cooperative effect of $n$ phonons depends on the spin-phonon coupling coefficients, $V_{n-\mathrm{ph}}$, and the Fourier transform of the $n$ phonon correlation function, $G^{n-\mathrm{ph}}$,

$$
\tau^{-1} \propto V_{n-\mathrm{ph}}^{2} G^{n-\mathrm{ph}}=\left(\frac{\partial^{n} \mathbf{V}}{\partial Q_{1} \ldots \partial Q_{n}}\right)^{2} G^{n-\mathrm{ph}},
$$

where $\mathbf{V}$ can be any of the tensors entering in the definition of $\hat{H}_{\mathrm{s}}$ and $G^{n-\mathrm{ph}}$ contains the phonons thermal population, $\bar{n}_{\alpha \mathbf{q}}$, and a condition imposing energy conservation. 


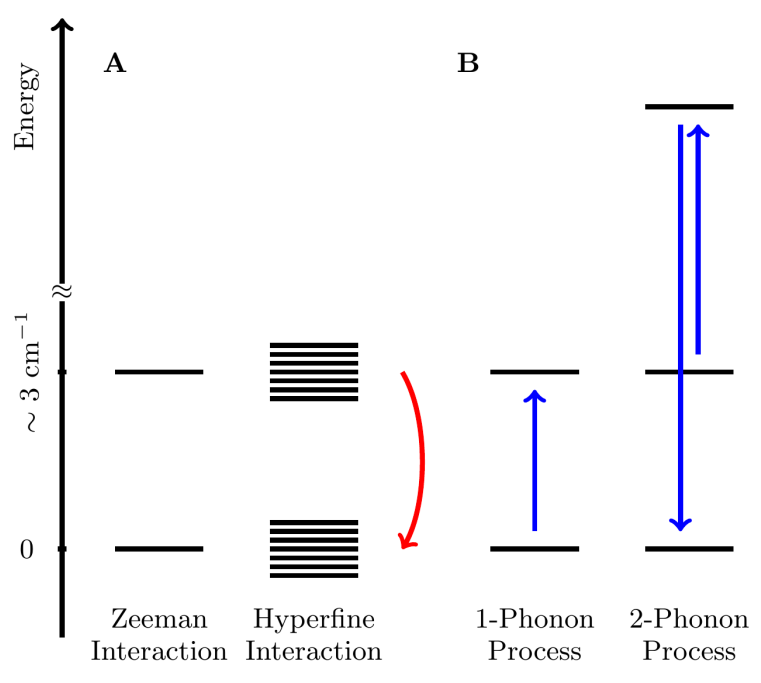

FIG. 1. Schematic Representation of Spin Energy Levels and Spin-Phonon Coupling Processes. (A) The effect of the Zeeman and hyperfine interaction on the spin energy levels with an external field of $\sim 3 \mathrm{~T}$. (B) An electronic spin-flip from an excited state to the ground state can be accompanied by the emission of a phonon with energy in resonance with the spin transition (one-phonon process) or the simultaneous absorption and emission of two high-energy phonons, whose energy difference is in resonance with the spin transition (two-phonon process).

Here we consider one- and two-phonon processes at the first-order of perturbation theory. One-phonon processes account for direct spin relaxation and contribute to the relaxation rate through $V_{1-\mathrm{ph}}=\left(\partial \mathbf{A} / \partial Q_{\alpha \mathbf{q}}\right)$ or $V_{1-\mathrm{ph}}=$ $\left(\partial \mathbf{g}_{\mathbf{e}} / \partial Q_{\alpha \mathbf{q}}\right)$, and $G^{1-p h}\left(\omega, \omega_{\alpha \mathbf{q}}\right) \propto \delta\left(\omega-\omega_{\alpha \mathbf{q}}\right) \bar{n}_{\alpha \mathbf{q}}+\delta(\omega+$ $\left.\omega_{\alpha \mathbf{q}}\right)\left(\bar{n}_{\alpha \mathbf{q}}+1\right)$. The first (second) term of $G^{1-p h}$ describes spin transitions involving the absorption (emission) of a single phonon from (to) the thermal bath. Figure $1 \mathrm{~B}$ schematically describe the emission process. Twophonon contributions, instead, involve the simultaneous modulation of the spin Hamiltonian by two phonons and open up Raman relaxation pathways. The relaxation rate due to simultaneous absorption/emission of two phonons, also depicted in Fig. $1 \mathrm{~B}$, depends on secondorder spin-phonon coupling coefficients, i.e. $V_{2-\mathrm{ph}}=$ $\left(\partial^{2} \mathbf{A} / \partial Q_{\alpha \mathbf{q}} \partial Q_{\beta \mathbf{q}^{\prime}}\right)$ or $V_{2-\mathrm{ph}}=\left(\partial^{2} \mathbf{g}_{\mathbf{e}} / \partial Q_{\alpha \mathbf{q}} \partial Q_{\beta \mathbf{q}^{\prime}}\right)$, and $G^{2-p h}\left(\omega, \omega_{\alpha \mathbf{q}}, \omega_{\beta \mathbf{q}^{\prime}}\right) \propto \delta\left(\omega-\omega_{\alpha \mathbf{q}}+\omega_{\beta \mathbf{q}^{\prime}}\right) \bar{n}_{\alpha \mathbf{q}}\left(\bar{n}_{\beta \mathbf{q}^{\prime}}+1\right)$.

$G^{1-\mathrm{ph}}$ has been investigated previously [11] starting from the force constants of the $3 \times 3 \times 3$ super-cell in Fig. $2 \mathrm{~A}$ calculated with density functional theory (DFT). At the $\mathrm{VO}(\mathrm{acac})_{2}$ characteristic frequencies the $\mathrm{V}$ spin is in resonance with acoustic phonons. These modes have a tiny phonon density of state (DOS), as their $\mathbf{q}$ approaches the $\Gamma$-point, but, at the same time, possess a large thermal population due to their low-energy $\left(\omega_{\alpha \mathbf{q}} \ll k_{\mathrm{B}} T\right)$. In order to obtain similar insights into Raman-driven relaxation, we use the DFT force constants and analyse
$\bar{G}^{2-\mathrm{ph}}=\sum_{\mathbf{q q}^{\prime}} \delta\left(\omega-\omega_{\alpha \mathbf{q}}+\omega_{\beta \mathbf{q}^{\prime}}\right) n_{\alpha \mathbf{q}}\left(n_{\beta \mathbf{q}^{\prime}}+1\right)$, where the integration is over the Brillouin zone. Fig. $2 \mathrm{C}$ shows $\bar{G}^{2-\mathrm{ph}}$ as function of both $\omega=\omega_{\alpha}-\omega_{\beta}$ and $\omega_{\beta}$. The maximum of this distribution shows the range of phonon energies, $\omega_{\beta}$, having a predominant contribution to the two-phonon DOS for a given value of $\omega$, and helps individuating the region of the phonons' spectrum responsible for the spin relaxation. The maximum contribution to the low-energy $\bar{G}^{2-p h}$ for $\omega$ close to the energy of available spin transitions, is provided by absorption/emission of phonons at $\sim 40 \mathrm{~cm}^{-1}$ with virtually no effect from phonons above $70 \mathrm{~cm}^{-1}$ and below $20 \mathrm{~cm}^{-1}$. Thus, while in principles the entire vibrational spectrum contributes to $\bar{G}^{2-\text { ph }}$, only a portion of the phonon's spectrum has, at the same time, a large DOS and thermal population.

In order to determine the spin-relaxation rate, associated to one- and two-phonon processes, we compute the corresponding spin-phonon coupling coefficients with supervised machine learning (ML). Our ML method consists in encoding the molecular structure into atomic-environment fingerprints, the bispectrum components [23, and use them together with Ridge regression to predict the spin-Hamiltonian coefficients [12, 13] (see Fig. 2B). Less than 2000 DFT reference data are enough to successfully train a ML model able to predict the eigenvalues of the $\mathbf{g}_{\mathbf{e}}$ and $\mathbf{A}$ tensors with a statistical error of only $\sim 2 \cdot 10^{-5}$ units and $\sim 4.2 \cdot 10^{-2} \mathrm{MHz}$, respectively. Once the ML model is trained, it is possible to rapidly scan $\mathbf{A}$ and $\mathbf{g}_{\mathbf{e}}$ along every molecular degrees of freedom and take the first- and second-order numerical derivatives at a negligible computational cost. An example of twodimensional scans of $\mathbf{A}$ and $\mathbf{g}_{\mathbf{e}}$ is reported in Fig. 2D. The relatively flat surface of the spin-Hamiltonian parameters nicely shows the appropriateness of the weak spin-phonon coupling regime assumed by the Redfield theory, a fact never demonstrated before. We stress that the secondorder numerical differentiation of $\mathbf{A}$ and $\mathbf{g}_{\mathbf{e}}$ requires the prediction of the spin-Hamiltonian coefficients for over $10^{5}$ molecular geometries. This volume of calculations is hardly sustainable with ab-initio methods, but becomes feasible on a large scale with ML.

Fig. 3A reports the spin-relaxation time $\tau$ due to twophonon excitations as a function of the external magnetic field and temperature. The hyperfine interaction is responsible for driving Raman relaxation at $B<1 \mathrm{~T}$, regardless of the temperature range, while Zeeman interaction is effective at higher fields. Figs. $3 \mathrm{~B}$ and $\mathrm{C}$ present a comparison between $\tau$ due to direct and Raman mechanisms at constant temperature, $T=20 \mathrm{~K}$, and constant field, $|B|=5 \mathrm{~T}$. Raman spin relaxation presents a $B^{-2}$ dependence at fields where the Zeeman interaction mediates the relaxation, while it becomes field independent at lower fields, where hyperfine interaction dominates. At the relatively high temperature of $20 \mathrm{~K}$, Raman relaxation dominates over the entire field window investigated. Nevertheless, the direct relaxation due to Zeeman interaction has a $B^{-4}$ field dependence and it is expected to take over the Raman mechanism at larger fields. At tem- 


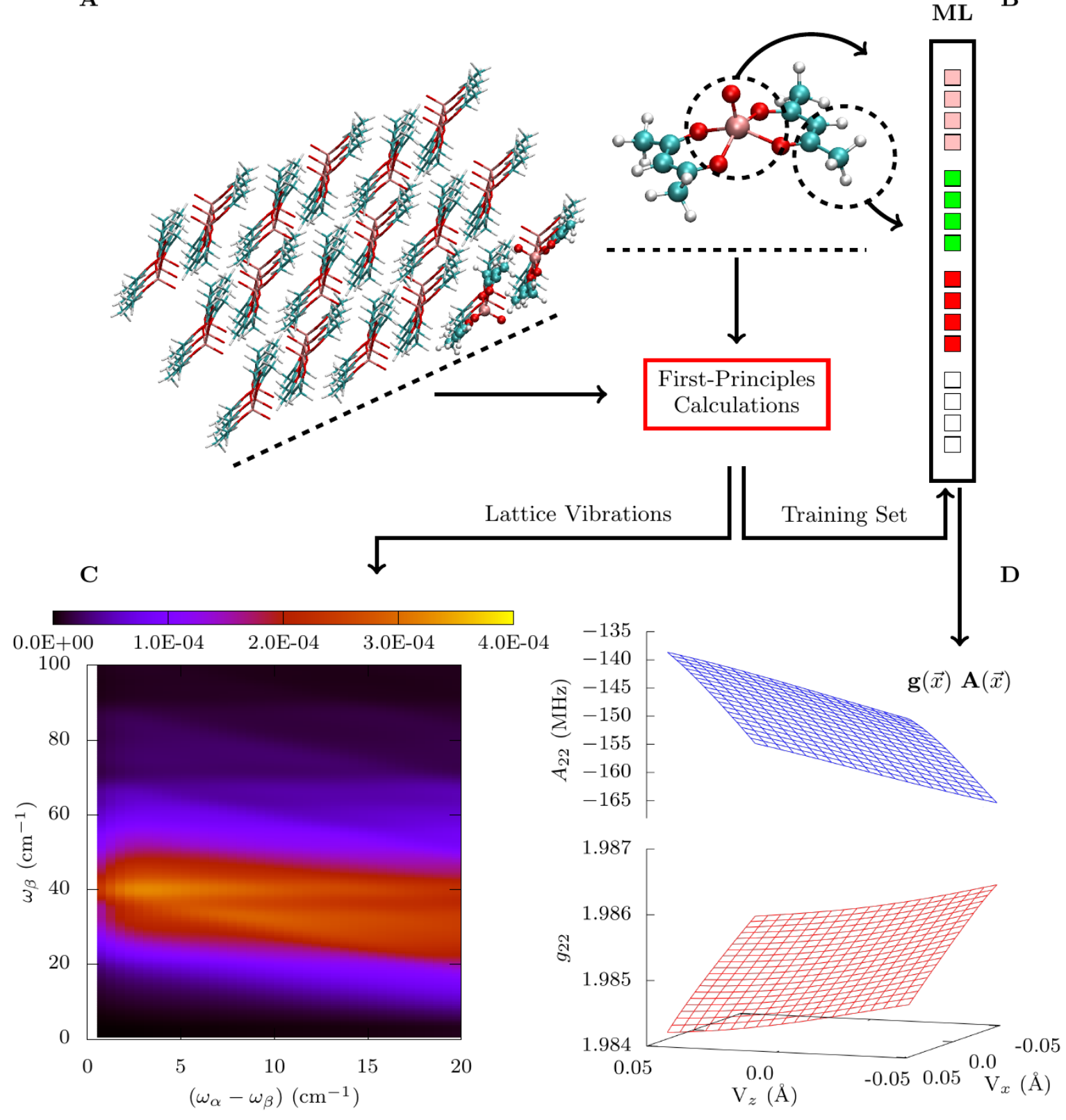

FIG. 2. First-Principles and ML Approach to Lattice and Spin Dynamics. (A) The $3 \times 3 \times 3$ replica of the VO(acac $)_{2}$ primitive cell used for the simulation of the crystal's vibrational properties and the structure of the isolated molecular unit used to generate the training set for the ML algorithm. (B) The schematic structure of the ML algorithm used to predict the magnetic properties as a function of the general atomic displacements. Each atomic environment is converted into a vector of fingerprints that determine the atomic contributions to the $\mathbf{A}$ and $\mathbf{g}_{\mathbf{e}}$ tensors. (C) The Fourier transform of the two-phonon correlation function, $G^{2-\mathrm{ph}}$, integrated over the Brillouin zone. (D) Examples of ML predictions for the hyperfine and Landè tensors as function of the $\mathrm{V}$ atomic displacements along $x$ and $z$.

peratures below $40 \mathrm{~K}$ Raman relaxation becomes exponentially slow due to the lack of thermal phonons and the direct relaxation mechanism takes over with a $T^{-1}$ dependence. In the ultra-low $T$ regime spin relaxation becomes independent of $T$ and proceeds through the spontaneous emission of a single phonon from an excited spin state. Finally, for $T>40 K, \tau$ reaches a $T^{-2}$ dependence.

Figures $3 \mathrm{~B}$ and $3 \mathrm{C}$ also report the comparison with AC experimental data [14. A good agreement is observed for high- $T$ high-field conditions, where the dipolar interactions are less effective or absent. Since experiments on magnetically diluted samples are not avail- 
A

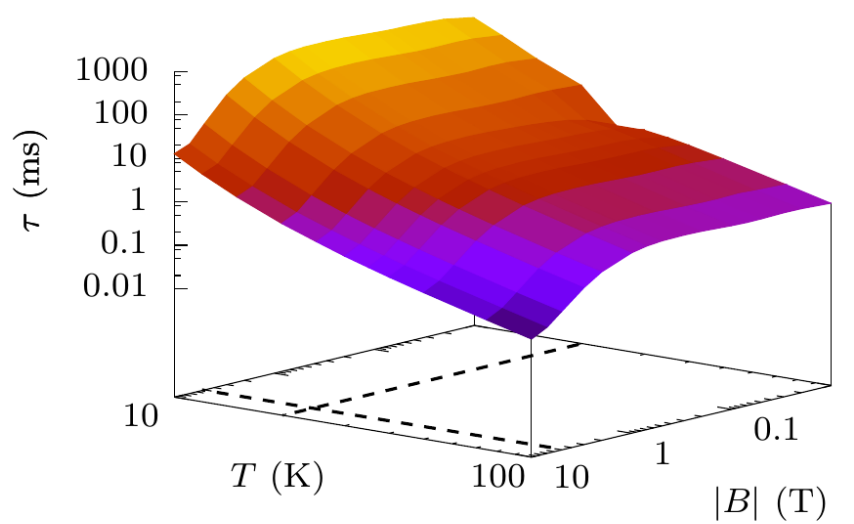

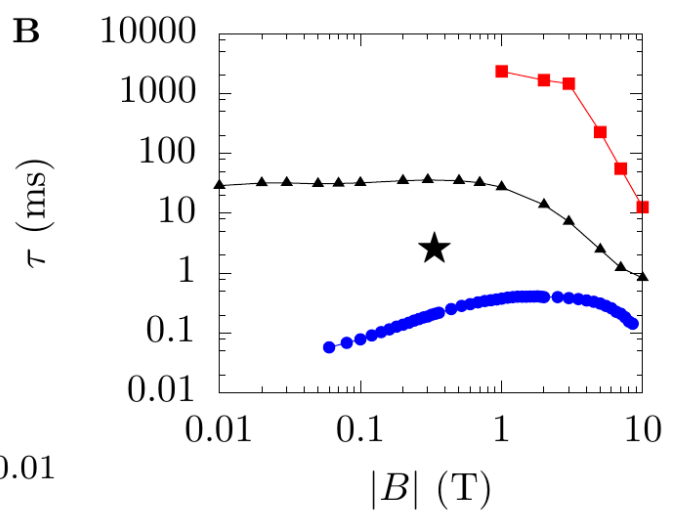

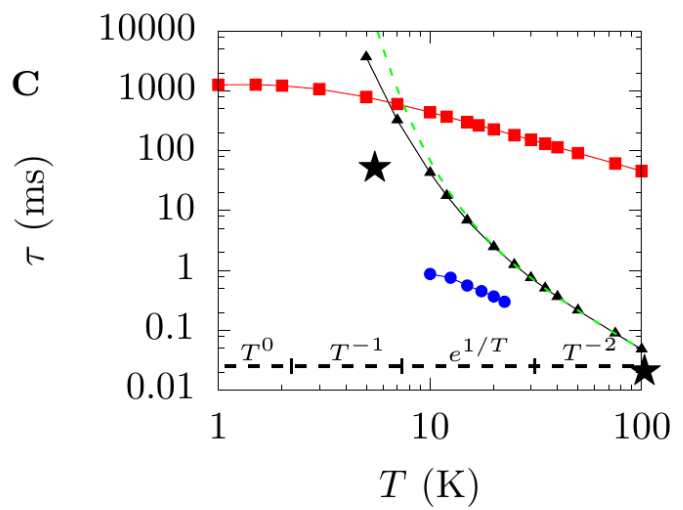

FIG. 3. Spin-phonon relaxation time. (A) Spin lifetime due to Raman relaxation as a function of the external magnetic field, $|B|$, and the temperature, $T$. The dashed black lines on the $B T$ plane are the path used for the one-dimensional plots reported in panels $(\mathbf{B}, \mathbf{C})$. (B) Computed direct (red line and squares), computed Raman (black line and triangles) and AC measured (blue line and circles) spin-relaxation times as function of the external magnetic field $|B|$ at $20 \mathrm{~K}$. The star corresponds to the average $T_{1}$ obtained from EPR measurements of magnetically diluted samples, both solid-state and frozen solution [1922. (C) Computed direct (red line and squares), computed Raman (black line and triangles) and AC measured (blue line and circles) spin-relaxation times as function of the temperature $T$ at an external magnetic field of 5 T. The star corresponds to the average $T_{1}$ obtained from X-Band EPR measurements of diluted samples, both solid-state and frozen solution 19 . 22 . The dashed green line corresponds to the fit of the second term of Eq. 3 . The dashed black line and labels help individuating the different $T$ regimes. (D) Cartesian displacements associated with the first and second optical modes at the $\Gamma$-point. The black structure is the $\mathrm{VO}(\mathrm{acac})_{2}$ equilibrium geometry as obtained from periodic-DFT optimization.

able for $\mathrm{VO}(\mathrm{acac})_{2}$, we compare our simulations with the $T_{1}$ obtained from electron paramagnetic resonance (EPR) on magnetically diluted samples of other Vanadyl compounds [1922]. All these molecules show relaxation times of the same order of magnitude across the temperature range 5-100 K. A representative value for their relaxation time is reported in Fig. 3B and Fig. 3C. Overall we observe a nice agreement between the simulated and experimental behaviour for this class of molecular qubits. The overestimation of the experimental results of approximately one order of magnitude is consistent with our overestimation of the vibrational frequencies and the underestimations of the hyperfine constants [11. We expect that further optimization of our first-principles methods and the inclusion of dipolar interactions will be able to further improve the agreement with experiments.

The temperature dependence of the spin-relaxation rate can be modelled with the expression,

$$
\tau^{-1}=V_{1-\mathrm{ph}} \frac{e^{\beta \omega_{1-\mathrm{ph}}}}{\left(e^{\beta \omega_{1-\mathrm{ph}}}-1\right)}+V_{2-\mathrm{ph}} \frac{e^{\beta \omega_{2-\mathrm{ph}}}}{\left(e^{\beta \omega_{2-\mathrm{ph}}}-1\right)^{2}},
$$

where $\beta=\left(k_{\mathrm{B}} T\right)^{-1}$. The second term is suggested by the product $\bar{n}_{\alpha \mathbf{q}}\left(\bar{n}_{\beta \mathbf{q}^{\prime}}+1\right)$ present in $G^{2-\text { ph }}$ under the condition $\bar{n}_{\alpha \mathbf{q}} \sim \bar{n}_{\beta \mathbf{q}^{\prime}}$ and can be used to identify the phonons responsible for Raman relaxation. A regression of the Raman relaxation time with the second term of Eq. (3), using $V_{2-\text { ph }}$ and $\omega_{2-\text { ph }}$ as free parameters, returns $\omega_{2-\mathrm{ph}} \sim 44 \mathrm{~cm}^{-1}$ for the dynamics at $|B|=5 \mathrm{~T}$, in nice agreement with the analysis done for $\bar{G}^{2-\mathrm{ph}}$. 
Phonons at such frequency are found across the entire Brillouin zone and are reminiscent of the first few $\Gamma$-point modes. The molecular distortions associated to the first two optical modes at the $\Gamma$-point are reported in Fig. $3 \mathrm{D}$ and show a strong contribution from Methyl rotations and from twisting of the acac ligands with respect to the Vanadyl unit.

These findings complement the significant experimental efforts that have been devoted to the study of the relation between vibrations and spin relaxation in recent years [7, 22, 24, 25] and conclusively point to lowenergy vibrations and soft intra-molecular modes as key to understand both Direct and Raman spin relaxation in molecular qubits.

The understanding of spin relaxation revealed by our first-principles calculations is rather different from the canonical Raman models, where the relaxation arises from uniform coupling of the spin to Debye-like acoustic modes across the entire Brillouin zone. This is expected to follow a $T^{-9}$ power law [26]. Such $T$-dependence is consistently not observed in molecular systems in favour of a $T^{-n}$ power law with $n$ often in the range 2-4 at high$T$ 27. Here we reproduce such behaviour and we reinterpret the origin of Raman spin-phonon relaxation as due to the modulation of magnetic interactions in the $\mathrm{MHz}-$ $\mathrm{GHz}$ energy range by a group of $\mathrm{THz}$ intra-molecular vibrations. These results thus support Klemens' interpretation of Raman relaxation as due to local vibrations
28. In addition it offers a new mean to interpret the existing vast spin-relaxation phenomenology in magnetic molecules and possibly improve their properties.

Taking a look at other solid-state spin qubits, such as organic radicals 29, solid-state defects [7, 30 and atoms adsorbed on surfaces 31, we note that they all likely present local vibrations with energy in the $\mathrm{THz}$ window able to modulate local magnetic interactions and initiate spin relaxation. Therefore we expect that the analysis offered here for $\mathrm{VO}(\mathrm{acac})_{2}$ also teaches important lessons for these systems. Finally, from a methodological point of view, given the recent great advancements of electronic structure methods [32] and ML for materials science 33], the delivery of an accurate and feasible computational approach to the prediction of spin-relaxation time in general classes of materials seems within the reach of the approach proposed here.

\section{Acknowledgements}

This work has been sponsored by AMBER (grant 12/RC/2278_P2). Computational resources were provided by the Trinity Centre for High Performance Computing (TCHPC) and the Irish Centre for High-End Computing (ICHEC). We also acknowledge the MOLSPIN COST action CA15128.

\section{Conflict of interests}

The authors declare no competing interests.
[1] Klaus Hornberger, Anton Zeilinger, and Markus Arndt, "Decoherence of matter waves by thermal emission of radiation," Nature 427, 711-714 (2004).

[2] David D Awschalom, Lee C Basset, Andrew S Dzurak, Evelyn $\mathrm{L} \mathrm{Hu}$, and Jason R Petta, "Quantum Spintronics: Engineering and Manipulating Atom-Like Spins in Semiconductors," Science 339, 1174-1179 (2013).

[3] Wolfgang Wernsdorfer and Mario Ruben, "Synthetic Hilbert Space Engineering of Molecular Qudits: Isotopologue Chemistry," Adv. Mater. 31, 1806687 (2019).

[4] Joseph M. Zadrozny, Jens Niklas, Oleg G. Poluektov, and Danna E. Freedman, "Millisecond Coherence Time in a Tunable Molecular Electronic Spin Qubit," ACS Cent. Sci. 1, 488-492 (2015)

[5] Muhandis Shiddiq, Dorsa Komijani, Yan Duan, Alejandro Gaita-Ariño, Eugenio Coronado, and Stephen Hill, "Enhancing coherence in molecular spin qubits via atomic clock transitions," Nature 531, 348-351 (2016)

[6] Sebastian F Maehrlein, Ilie Radu, Pablo Maldonado, Alexander Paarmann, Michael Gensch, Alexandra M Kalashnikova, Roman V Pisarev, Martin Wolf, Peter M Oppeneer, Joseph Barker, and Tobias Kampfrath, "Dissecting spin-phonon equilibration in ferrimagnetic insulators by ultrafast lattice excitation," Sci. Adv. 4, eaar5164 (2018).

[7] T. Astner, J. Gugler, A. Angerer, S. Wald, S. Putz, N. J. Mauser, M. Trupke, H. Sumiya, S. Onoda, J. Isoya, J. Schmiedmayer, P. Mohn, and J. Majer, "Solidstate electron spin lifetime limited by phononic vacuum modes," Nat. Mater. 17, 313-317 (2018).

[8] Peter Caravan, "Strategies for increasing the sensitivity of gadolinium based MRI contrast agents," Chem. Soc. Rev 35, 512-523 (2006).

[9] L. Escalera-Moreno, N. Suaud, A. Gaita-Ariño, and E. Coronado, "Determining Key Local Vibrations in the Relaxation of Molecular Spin Qubits and Single-Molecule Magnets," J. Phys. Chem. Lett. 8, 1695-1700 (2017)

[10] Conrad A.P. Goodwin, Fabrizio Ortu, Daniel Reta, Nicholas F. Chilton, and David P. Mills, "Molecular magnetic hysteresis at 60 kelvin in dysprosocenium," $\mathrm{Na}-$ ture 548, 439-442 (2017)

[11] Alessandro Lunghi and Stefano Sanvito, "How do phonons relax molecular spins?" Sci. Adv. 5, eaax7163 (2019).

[12] Alessandro Lunghi and Stefano Sanvito, "A unified picture of the covalent bond within quantum-accurate force fields : From organic molecules to metallic complexes ' reactivity," Sci. Adv. 5, eaaw2210 (2019).

[13] Alessandro Lunghi and Stefano Sanvito, "Surfing Multiple Conformation-Property Landscapes via Machine Learning: Designing Single-Ion Magnetic Anisotropy," J. Phys. Chem. C 124, 5802-5806 (2020).

[14] Lorenzo Tesi, Alessandro Lunghi, Matteo Atzori, Eva Lucaccini, Lorenzo Sorace, Federico Totti, and Roberta Sessoli, "Giant spin-phonon bottleneck effects in evaporable vanadyl-based molecules with long spin coherence," Dalt. Trans. 45, 16635-16645 (2016)

[15] L Childress, M V Gurudev Dutt, J M Taylor, A S Zibrov, 
F Jelezko, J Wrachtrup, P R Hemmer, and M D Lukin, "Coherent Dynamics of Coupled Electron and Nuclear Spin Qubits in Diamond," Science 314, 281-286 (2006).

[16] Juha T Muhonen, Juan P Dehollain, Arne Laucht, Fay E Hudson, Rachpon Kalra, Takeharu Sekiguchi, Kohei M Itoh, David N Jamieson, Jeffrey C McCallum, Andrew S Dzurak, and Andrea Morello, "Storing quantum information for 30 seconds in a nanoelectronic device," Nat. Nanotechnol. 9, 986 (2014).

[17] C Godfrin, A Ferhat, R Ballou, S Klyatskaya, M Ruben, W Wernsdorfer, and F Balestro, "Operating Quantum States in Single Magnetic Molecules: Implementation of Grover's Quantum Algorithm," Phys. Rev. Lett. 119, 187702 (2017).

[18] A G Redfield, Adv. Magn. Opt. Reson., Vol. 1 (Academic Press Inc., 1965) pp. 1-32.

[19] Matteo Atzori, Lorenzo Tesi, Elena Morra, Mario Chiesa, Lorenzo Sorace, and Roberta Sessoli, "RoomTemperature Quantum Coherence and Rabi Oscillations in Vanadyl Phthalocyanine: Toward Multifunctional Molecular Spin Qubits," J. Am. Chem. Soc. 138, 21542157 (2016)

[20] Chung Jui Yu, Michael J. Graham, Joseph M. Zadrozny, Jens Niklas, Matthew D. Krzyaniak, Michael R. Wasielewski, Oleg G. Poluektov, and Danna E. Freedman, "Long Coherence Times in Nuclear Spin-Free Vanadyl Qubits," J. Am. Chem. Soc. 138, 14678-14685 (2016)

[21] Matteo Atzori, Elena Morra, Lorenzo Tesi, Andrea Albino, Mario Chiesa, Lorenzo Sorace, and Roberta Sessoli, "Quantum Coherence Times Enhancement in Vanadium(IV)-based Potential Molecular Qubits: The Key Role of the Vanadyl Moiety," J. Am. Chem. Soc. 138, 11234-11244 (2016)

[22] Matteo Atzori, Stefano Benci, Elena Morra, Lorenzo Tesi, Mario Chiesa, Renato Torre, Lorenzo Sorace, and Roberta Sessoli, "Structural Effects on the Spin Dynamics of Potential Molecular Qubits," Inorg. Chem. 57, 731740 (2018)

[23] Albert P. Bartok, Risi Kondor, and Gabor Csanyi, "On representing chemical environments," Phys. Rev. B 87, 184115 (2013)

[24] Duncan H. Moseley, Shelby E. Stavretis, Komalavalli Thirunavukkuarasu, Mykhaylo Ozerov, Yongqiang Cheng, Luke L. Daemen, Jonathan Ludwig, Zhengguang Lu, Dmitry Smirnov, Craig M. Brown, Anup Pandey, A. J. Ramirez-Cuesta, Adam C. Lamb, Mihail Atanasov, Eckhard Bill, Frank Neese, and Zi Ling Xue, "Spinphonon couplings in transition metal complexes with slow magnetic relaxation," Nat. Commun. 9, 2572 (2018)

[25] E Garlatti, L Tesi, A Lunghi, M Atzori, D J Voneshen, P Santini, S Sanvito, T Guidi, R Sessoli, and S Carretta, "Unveiling phonons in a molecular qubit with four-dimensional inelastic neutron scattering and density functional theory," Nat. Commun. , DOI:0.1038/s41467020-15475-7 (2020).

[26] K N Shrivastava, "Theory of Spin-Lattice Relaxation," Phys. Status Solidi 117, 437-458 (1983).

[27] Ana-Maria Ariciu, David H Woen, Daniel N Huh, Lydia E Nodaraki, Andreas K Kostopoulos, Conrad A P Goodwin, Nicholas F Chilton, Eric J L Mcinnes, Richard E P Winpenny, William J Evans, and Floriana Tuna, "Engineering electronic structure to prolong relaxation times in molecular qubits by minimising orbital angular momentum," Nat. Commun. 10, 3330 (2019)

[28] P. G. Klemens, "Localized modes and spin-lattice interactions," Phys. Rev. 125, 1795-1798 (1962).

[29] Sam Schott, Erik R McNellis, Christian B Nielsen, HungYang Chen, Shun Watanabe, Hisaaki Tanaka, Iain McCulloch, Kazuo Takimiya, Jairo Sinova, and Henning Sirringhaus, "Tuning the effective spin-orbit coupling in molecular semiconductors," Nat. Commun. 8, 15200 (2017)

[30] Federico Lombardi, Alessandro Lodi, Ji Ma, Junzhi Liu, Michael Slota, Akimitsu Narita, William K Myers, Klaus Müllen, Xinliang Feng, and Lapo Bogani, "Quantum units from the topological engineering of molecular graphenoids," Science 366, 1107-1110 (2019).

[31] Kai Yang, William Paul, Soo-hyon Phark, Philip Willke, Yujeong Bae, Taeyoung Choi, Taner Esat, Arzhang Ardavan, Andreas J Heinrich, and Christopher P Lutz, "Coherent spin manipulation of individual atoms on a surface," Science 366, 509-512 (2019).

[32] Frank Neese, Mihail Atanasov, Giovanni Bistoni, Dimitrios Manganas, and Shengfa Ye, "Chemistry and Quantum Mechanics in 2019 - Give us Insight and Numbers," J. Am. Chem. Soc. 141, 2814-2824 (2019).

[33] Keith T. Butler, Daniel W. Davies, Hugh Cartwright, Olexandr Isayev, and Aron Walsh, "Machine learning for molecular and materials science," Nature 559, 547555 (2018). 